\title{
Gene silencing in Xenopus laevis by DNA vector-based RNA interference and transgenesis
}

\author{
Ming $\mathrm{Li}^{1}$, Baerbel Rohrer ${ }^{1,2}$ \\ Departments of ${ }^{1}$ Neurosciences and ${ }^{2}$ Ophthalmology, Division of Research, Medical University of South Carolina, 167 Ashley Ave, \\ SEI 511, Charleston, SC 29425, USA
}

A vector-based RNAi expression system was developed using the Xenopus tropicalis U6 promoter, which transcribes small RNA genes by RNA polymerase III. The system was first validated in a Xenopus laevis cell line, designing a short hairpin DNA specific for the GFP gene. Co-transfection of the vector-based RNAi and the GFP gene into Xenopus XR1 cells significantly decreased the number of GFP-expressing cells and overall GFP fluorescence. Vector-based RNAi was subsequently validated in GFP transgenic Xenopus embryos. Sperm nuclei from GFP transgenic males and RNAi construct-incubated-sperm nuclei were used for fertilization, respectively. GFP mRNA and protein were reduced by $\sim 60 \%$ by RNAi in these transgenic embryos compared with the control. This transgene-driven RNAi is specific and stable in inhibiting GFP expression in the Xenopus laevis transgenic line. Gene silencing by vector-based RNAi and Xenopus transgenesis may provide an alternative for 'repression of gene function' studies in vertebrate model systems.

Cell Research (2006) 16:99-105. doi:10.1038/sj.cr.7310013; published online 16 January 2006

Keywords: RNAi, Xenopus U6 promoter, transgenic, Xenopus laevis, GFP

\section{Introduction}

Amphibians have been widely used as a vertebrate model for studying early development. Xenopus is a popular model due to the ready availability of oocytes and embryos in large quantities and the easy accessibility for cell and tissue manipulation. Studies with Xenopus have been used to elucidate the molecular mechanisms and signaling of pattern formation, differentiation, as well as processes of diseases which are general to all vertebrates [1-3]. Gene manipulations have long been used to study gene function in Xenopus. Ectopic expression of injected mRNA has been used effectively to study maternally-expressed genes. Dominant negative mutants, antisense oligonucleotides and ribozymes are the approaches to suppress specific gene expression for functional studies [4]. The limitations, such

Correspondence: Baerbel Rohrer

Tel: +843-792-5086; Fax: +843-792-1723;

E-mail: rohrer@musc.edu

Received 26 Sep 2005; revised 27 October 2005; accepted 29 October 2005; published online 16 January 2006 as the lack of control over the spatial and temporal effects of these transient expression studies, were overcome by Kroll and Amaya in 1996 [5]. In their system, linearized plasmid DNA is introduced into demembranated and decondensed sperm nuclei using restriction enzyme-mediated integration (REMI), and the nuclei are injected into unfertilized eggs. This procedure produces hundreds of embryos that express the integrated plasmids non-mosaically. The establishment of transgenic frog lines was a breakthrough to study spatial and temporal gene expression and revived Xenopus as one of the favorite models for functional genomics $[6,7]$. The combination of this transgenesis approach together with dominant negative mutations, or ribozyme and antisense technology, allows functional 'knockdown' of specific genes for 'loss-of-function' studies.

A major challenge in present day biological studies is to decipher the molecular function of thousands of genes. Knockout transgenic mice are very useful, but laborious and expensive. Double-stranded RNA (dsRNA) induces genespecific silencing in living organisms from fungi to animals, a phenomenon named RNA interference (RNAi). RNAi, a rapidly developing technique, has shown great promise in 
depressing gene expression in a variety of systems from C. elegans to plants and animals $[8,9]$. Small interfering RNA duplexes (siRNA) 19 - 23 nucleotides in length, synthesized chemically or in vitro transcribed are used to knock down target genes. Most studies have been done in mammalian cells or mice. Recently, DNA expression vector-based or PCR-based siRNA has been developed. In these experiments, a short hairpin RNA (shRNA) consisting of sense and antisense RNAs connected by a loop sequence are generated in cells, transcribed by either RNA polymerase II using CMV or by RNA polymerase III using the mouse U6 or mouse and human $\mathrm{H} 1$ promoters [10]. Retroviral or lentiviral vector-based siRNAs have been used to increase transfection efficiency or to integrate short hairpin DNAs into the genome $[11,12]$. Reports on the use of RNAi in Xenopus have been limited to microinjection of oocytes or early blastomeres with long or short dsRNA to study gene function in early stages $[13,14]$. The dsRNA in this method is present transiently and stable gene silencing in later stages is difficult to achieve. Stable transgenesis with DNA-based RNAi may overcome this difficulty when the expression cassette is integrated into the genome of the recipient.

In the present study, we have combined RNAi and transgenesis techniques, and introduced stable RNAi into Xenopus via transgenesis. Xenopus tropicalis U6-driven expression of small hairpin RNA directed against GFP was validated in cultured Xenopus cells in vitro and in GFP transgenic Xenopus embryos in vivo. Stable gene inhibition by transgene-driven RNAi may be practicable and provide a useful tool for genomic studies.

\section{Materials and methods}

\section{Plasmid constructs}

The $X$. tropicalis U6 promoter was amplified from the pUC19-U6 gene (a generous gift from Dr. Philippe Carbon, Institut de Biologie Moleculaire du CNRS, Strasbourg Cedex, France) [15] with the following primers: U6 forward, 5'-GGG AAT TCG AGG CCG TGT GAT AAA GG-3', and U6 reverse, 5'-GGA AGC TTG GTA CCG GAT CCA GCG AGC AGC ACC GCC TTA-3'. EcoR I, BamH I and Hind III restriction enzyme sites were introduced into the above primers. The PCR products were digested with EcoR I and Hind III and cloned into pUC19 to get the pUC19-U6 plasmid. To construct the hairpin siRNA expression cassette, two complementary DNA oligos, 5'-GAT CCG GcG ATG CcA CcT ACG GAA TTC AAG AGA TTC GTA gGT gGC ATC gCC T TT TTT GGAAA-3'; 5'-AGC TTT TCC AAAAAA GGc GAT GCc Acc TAC GGAATC TCT TGA ATT CCG Tag GTg GCA TCg CCG-3' (for EF- $1 \alpha-$ GFP in XIG), and 5'-GAT CCG GtG ATG CaA CaT ACG GAA TTC AAG AGA TTC CGT AtG TtG CAT CaC CTT TTT TGG AAA-3', 5'-AGC TTT TCC AAA AAA GGt GAT GCa ACa TAC GGA ATC TCT TGA ATT CCG Tat GAa GCA TCa CCG-3' (for CMV-GFP in transgenic Xenopus) were synthesized, annealed and inserted into the BamH IHind III site of pUC19-U6. The inserted sequences were verified by
DNA sequencing. The Xenopus expression plasmid XIG containing the EF-1 $\alpha$ promoter driving the expression of GFP was generously provided by Nancy Hopkins (Massachusetts Institute of Technology) [16]. Due to the differences in the GFP gene sequences indicated by lower bold letters, shRNA targeting GFP in the XIG construct has no effect on the expression of GFP in the transgenic frog line.

\section{Cell transfections}

Xenopus XR1 cells [17] were grown at room temperature in $60 \%$ L-15 medium supplemented with $10 \%$ fetal bovine serum and $1 \%$ embryo extract. Cells were plated on $12 \mathrm{~mm}$ glass coverslips in 35 $\mathrm{mm}$ dishes with $1.5 \mathrm{~mL}$ culture medium and grown to $60 \%$ confluency. The medium was changed to serum-reduced medium $(1 \%$ FBS) before transfection. A mixture of plasmids and DOTAP (Roche, Mannheim, Germany) at a molecular weight ratio of 1:7 was added into the dishes for $48 \mathrm{~h}$. The cells were then rinsed with PBS and fixed in $4 \%$ paraformaldehyde for $30 \mathrm{~min}$. The slides were coverslipped and analyzed for fluorescence using fluorescence microscopy (Zeiss). GFP positive cells were counted in 96 fields, each covering an area of 430 by $340 \mu \mathrm{m}$, from each group. Three independent transfections were compared for this study. Fluorescence on 25 randomly selected GFP positive cells were measured with Scion Image (Scion Corporation, Frederick, MD) software. Data were represented as the mean \pm standard deviation and analyzed using Student $t$ test.

\section{Transgenesis and Identification}

Transgenic Xenopus embryos were produced by restriction-enzyme-mediated integration (REMI) as described [5]. Briefly, sperm nuclei were isolated from the testes of male GFP transgenic frogs (kindly provided by EM De ROBERTIS, University of California, Los Angeles, and D BROWN, Carnegie Institution of Washington) [1]. Linearized plasmid DNA was introduced into decondensed sperm nuclei using REMI and the nuclei were transplanted into unfertilized eggs.

For fluorescence imaging, animals were anesthetized in $0.01 \%$ 3-aminobenzoic acid ethyl ester (Sigma). GFP expression was monitored using fluorescent microscopy at $4 \times$. The intense fluorescence of the tadpole lens permits rapid screening of potential transgenic animals. Definitive transgenesis was confirmed by PCR. Tadpole tail tip or another small piece of tissue was digested with $1 \mathrm{mg} / \mathrm{mL}$ proteinase $\mathrm{K}$ in PCR buffer for a few hours at $55^{\circ} \mathrm{C}$, boiled for 10 $\mathrm{min}$, and centrifuged briefly at $12,000 \mathrm{~g}$. The supernatant was used as a template for PCR, using specific primers designed against the plasmid: 5'-GGC ACT TTG GTT CCA TCT GT-3', 5'-GAG TCA GTG AGC GAG GAA GC-3'; or GFP: 5'-AAG GTG ATG CAA CAT ACG G-3' and 5'-ACA GGG CCA TCG CCA ATT G-3'. Older tadpoles were large enough that they could be used for more than two purposes (e.g., genotyping, eye sectioning, RNA or protein extractions).

\section{Histology}

Xenopus embryos and larvae were fixed in 4\% paraformaldehyde for $24 \mathrm{~h}$ and cryoprotected in $30 \%$ sucrose. The animals were frozen in TBS tissue freezing medium and sectioned at $18 \mu \mathrm{m}$ using a Cryostat (Microm). The sections were mounted on poly-L-lysine-coated slides and rinsed with phosphate buffered saline (PBS). Transverse sections through the eye were photographed using a digital camera (Spot) and the fluorescence intensity in the central retina was determined using Scion Image software. Data were represented as the mean \pm standard deviation and analyzed using Student $t$ test. 


\section{$R T-P C R$}

Total RNAs were isolated from individual genotyped embryo of different stages using Trizol reagent (Invitrogen). RNA $(1 \mu \mathrm{g})$ was used for reverse transcription using Superscript First-Strand Synthesis Kit (Invitrogen) at $42^{\circ} \mathrm{C}$ for one hour. Product $(1 \mu \mathrm{L})$ diluted 1:10 was used for 27 PCR cycles, which was determined to be within the linear amplification range for both transcripts. GFP-specific primers were 5'-AAGGTGATGCAACATACGG-3' and 5'-ACA GGG CCA TCG CCAATT G-3' (477 bp); Xenopus $\beta$-actin primers 5'-CCATTG GTA ACG AGC GTT T-3' and 5'-GAG GGG CCA GAC TCA TCA TA-3' (358 bp) were used as an internal control.

Quantitative real-time RT-PCR was done using the SYBR Green PCR Master Mix and an ABI Prism 7000 Sequence Detection System (Applied Biosystems, Foster City, CA). Quantitative values were obtained by determining the cycle number $\left(\mathrm{C}_{\mathrm{t}}\right.$ value $)$, at which a clearly detectable increase in fluorescence occurs, using the software provided by the system. All $\mathrm{C}_{\mathrm{t}}$ values were normalized to their corresponding $\beta$-actin levels.

\section{Western blot}

Individual genotyped embryos were homogenized with lysis buffer and $25 \mu \mathrm{g}$ of lysate protein was loaded onto a $10 \%$ SDS-PAGE gel and transferred to a Hybond ECL nitrocellulose membrane (Amershan Pharmacia). Rabbit anti-GFP (Santa Cruz) and goat anti-rabbit IgG-HRP conjugate (Santa Cruz) were used for detection, and ECL+ was used for visualization (Bio-Rad Imager). The blots were reprobed with anti- $\beta$-actin antibody (Santa Cruz) to control for loading.

\section{Results}

The goal of our research is to achieve stable inhibition of the expression of a specific gene in Xenopus, by introducing stable RNA interference via transgenesis. In this study, the $X$. tropicalis U6 gene promoter [15] was obtained to drive the expression of shRNA targeting GFP. The U6 promoterdriven hairpin type dsRNA construct was designed to contain the sense strand of the target (i.e., GFP, basepairs $104 \mathrm{bp}$ to $124 \mathrm{bp}$ ), a short spacer (TTCAAGAGA), and the antisense strand of the target (Figure 1A). To verify that silencing is conferred by the U6-shRNA construct, the siRNA expressing plasmid was co-transfected together with a GFP expressing plasmid, XIG [16], into Xenopus XR1 cells [17]. Vector-based RNAi was found to knock down the expression of GFP in Xenopus XR1 cells in vitro. Silencing of GFP was detected $48 \mathrm{~h}$ posttransfection in cells co-transfected with the U6-shRNA plasmid (Figure 1B), but not in cells with control plasmid, pUC19-U6 (Figure 1C). Not only did the overall number of cells expressing GFP decrease (Figure 1D), but the GFP fluorescence intensity of individual cells was found to be significantly weaker in GFP positive cells in the U6-shRNA transfected group compared to those from the control group $(P<0.05)$ (Figure $1 \mathrm{E})$. Taken together, these results showed first, that the GFP targeting sequence that had been proven to be effective in mammalian cells [10] could also be used in Xenopus cells; and second, that the Xenopus U6 promoter could be

A ShRNA

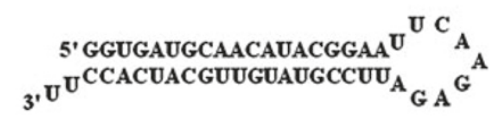

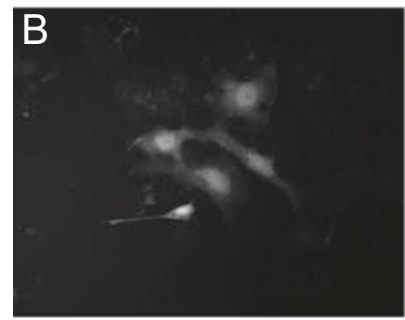

$\mathrm{XIG}+$ shRNA

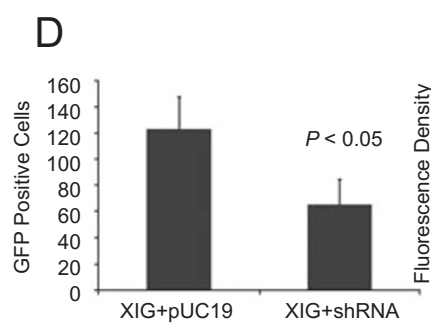

Figure 1 ShRNA directed against GFP knocks down the expression of the GFP gene in Xenopus XR1 cells. (A) Sequence and putative folding of the small hairpin RNA targeting the GFP gene. (B), (C) are fluorescence images of Xenopus XR1 cells transfected with XIG GFP plus pUC19/U6/shRNA, and XIG GFP plus pUC19/U6, respectively. (D) GFP positive cells were counted in 96 fields from each group. The average is from three independent transfections, $P<$ 0.05 . (E) Fluorometric measurement of GFP intensity was analyzed from 25 randomly selected GFP positive cells per condition. Data were expressed in arbitrary units and normalized to 100 in the control $(P<0.05)$. Scale bar in $\mathrm{C}=30 \mu \mathrm{m}$.

used effectively to express siRNA in the Xenopus system, just like the human H1, human U6 or mouse U6, which drive expression of siRNAs in their respective mammalian systems $[19,20]$. Since the transfection efficiency was low in this cell line (less than 15\%), further validation and confirmation of the GFP silencing and specificity was not performed in the cell model.

We went on to confirm that transgene-driven expression of shRNA knocked down GFP expression in GFP transgenic embryos in vivo. Sperm nuclei were prepared from GFP transgenic adult male frogs. In these frogs, GFP is driven by the simian CMV immediate early promoter [6]. First, it was established how many integration events have occurred in the founder frog that was used for the entire study [6]. Sperm nuclei were injected into unfertilized eggs and the progeny that expressed GFP was determined. Approximately $50 \%$ of the resulting progeny were found to be GFP positive, indicative of a single integration event. Since the GFP transgenic line contains one integration event and 
the CMV drives a strong expression, all the animals have similarly high GFP expression [6], which is advantageous when quantifying the effects of RNAi.

Restriction enzyme-mediated integration (REMI) transgenesis was performed in all subsequent experiments, as published by Kroll and Amaya [5, 21]. As described in that procedure, the control (pUC19) or the U6-shRNA plasmid DNA was integrated into the genomic DNA of the sperm nuclei by incubation with the respective linearized plasmid DNA, in the presence of a small amount of EcoR I restriction enzyme, and egg extract. These sperm nuclei were then transplanted into unfertilized eggs by microinjection. The viability of embryos generated by transgenesis (DNA incubated sperm nuclei) was lower than the embryos generated by sperm nuclei transplantation only, but no obvious difference in viability between the pUC19 and U6-shRNA transgenesis was observed. The embryos were examined for GFP fluorescence at 5 or 6 days of development (stage (St) $33-42$ ). Roughly $50 \%$ of the embryos generated with the control pUC19 were found to be GFP positive (101/193; embryos analyzed from 2 experiments). Likewise, the percentage of GFP positive embryos generated by the U6-shRNA transgenesis was $\sim 50 \%(110 / 218$; 2 experiments), which was not significantly different from the control. However, the two GFP positive populations differed significantly in their fluorescence intensity. Fluorescence was strong and evenly distributed in the majority of the embryos generated with pUC19, whereas the fluorescence in the U6-shRNA group was significantly weaker and the distribution was more variable (Figure 2). When fluorescence intensity of these embryos was scored on a scale from 3 to 1 (3: bright; 2 : average, 1 : low), the median fluorescence intensity was shifted from 3 (no plasmid or pUC19 transgenics) to 2 (U6-shRNA) (Table 4). This pool of embryos was not genotyped and therefore still contains U6-shRNA negative embryos. The decrease in the median fluorescence score is consistent with the decrease in GFP protein content obtained from 25 randomly chosen GFPpositive embryos compared to the controls $(\sim 30 \%)$.

Genotyping was performed on a subset of shRNA transgenic animals at stage 40 (St 40) to identify the double transgenic animals (U6-shRNA and GFP) by using PCR to correlate genotype with GFP-suppression (Table 2). U6-shRNA transgenesis was $\sim 40 \%$, which is consistent with XIG and other GFP reporter constructs we performed (data not shown). The GFP fluorescence was inhibited significantly in U6-shRNA positive embryos as shown by overall fluorescence (Figure 2A) or when analyzing a specific tissue such as the eye (Figure 3A), when compared with the shRNA negative embryos (Figure 2B) and tissues (Figure 3B). Fluorescence intensity measured in the central retina showed $\sim 50 \%$ reduction in GFP fluorescence

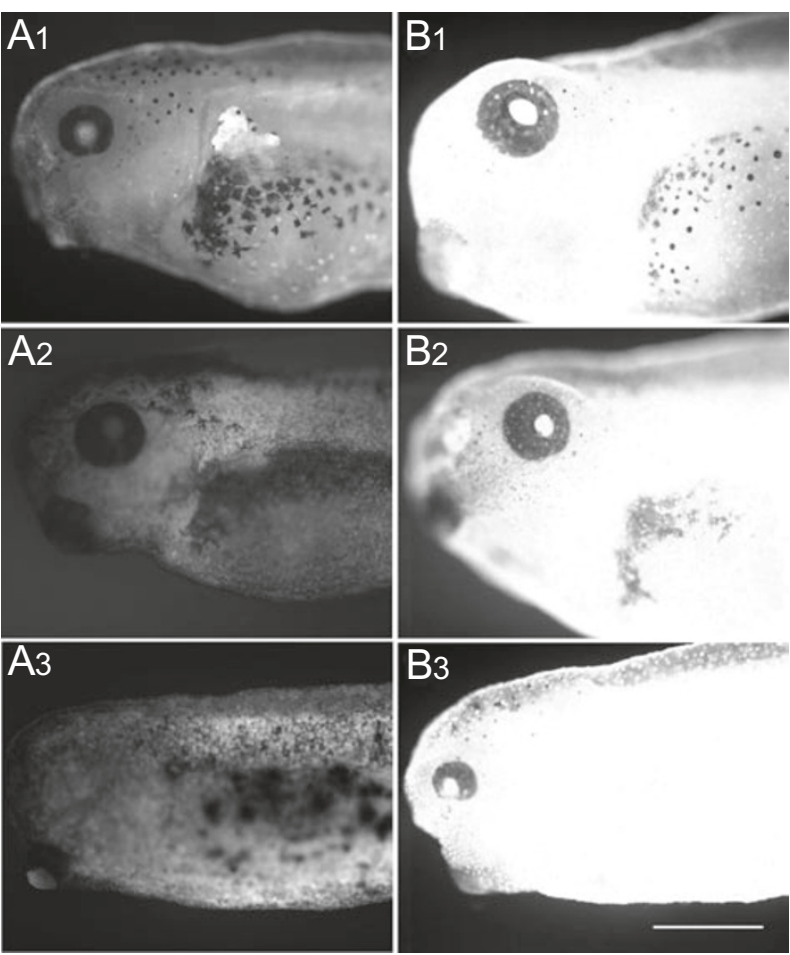

Figure 2 Transgene-driven RNAi knocks down GFP expression in GFP transgenic embryos. The animal images of shRNA transgenic embryos of St 39 to 41 (A1-A3) reveal the repression of GFP fluorescence compared with the control pUC19 tansgenics (B1-B3). Scale bar for embryos in $\mathrm{B} 3=500 \mu \mathrm{m}$

Table 1 GFP-intensity of transgenic embryos

\begin{tabular}{ccccc}
\hline GFP & +++ & ++ & + & Median \\
\hline shGFPs & $37 / 33.6 \%$ & $42 / 38.2 \%$ & $31 / 28.2 \%$ & 2 \\
& & & & \\
shGFPn & $54 / 73.0 \%$ & $19 / 25.7 \%$ & $1 / 1.4 \%$ & 3 \\
& & & & 3 \\
\hline
\end{tabular}

The transgenic embryos ( $\sim 100$ per condition; not genotyped) at stage 33-42 were examined at $4 \times$ under the fluorescence microscope and sorted according to the fluorescence intensity; strong $(+++$, three points), average ( ++ , two points), weak ( + , one point). The data are expressed as the number of embryos and their percentage of the total. Specific shGFP (shGFPs) shifted the median intensity from 3 to 2 when compared to the control plasmid (pUC19), whereas the mismatched or nonspecific shGFP (shGFPn) had no effect.

in the shRNA embryos (Figure 3C). Quantitative RT-PCR showed that relative GFP mRNA levels were reduced by $\sim 60 \%(P<0.01)$ in U6-shRNA transgenic embryos compared with control embryos (Figure 4A, 4C). Likewise, immunoblotting confirmed that GFP protein expression in 
Table 2 Genotyping of the transgenic embryos

\begin{tabular}{lccc}
\hline Genotyping & GFP- & GFP+ & Total \\
\hline SiRNA+ & $5 / 41.7 \%$ & $19 / 40.4 \%$ & $24 / 40.7 \%$ \\
SiRNA- & $7 / 58.3 \%$ & $28 / 59.6 \%$ & $35 / 59.3 \%$ \\
Total & $12 / 100 \%$ & $47 / 100 \%$ & $59 / 100 \%$ \\
\hline
\end{tabular}

GFP negative (GFP-; $n=12$ ) and GFP positive (GFP+; $n=47)$ animals were chosen at random and tested for (SiRNA + ) or absence (SiRNA-) of the transgene by PCR. Embryo number and percentage are listed. As expected, $\sim 40 \%$ of the tadpoles were found to be positive for the transgene. This population was used for histology, RNA and protein analysis (Figures 3 and 4).
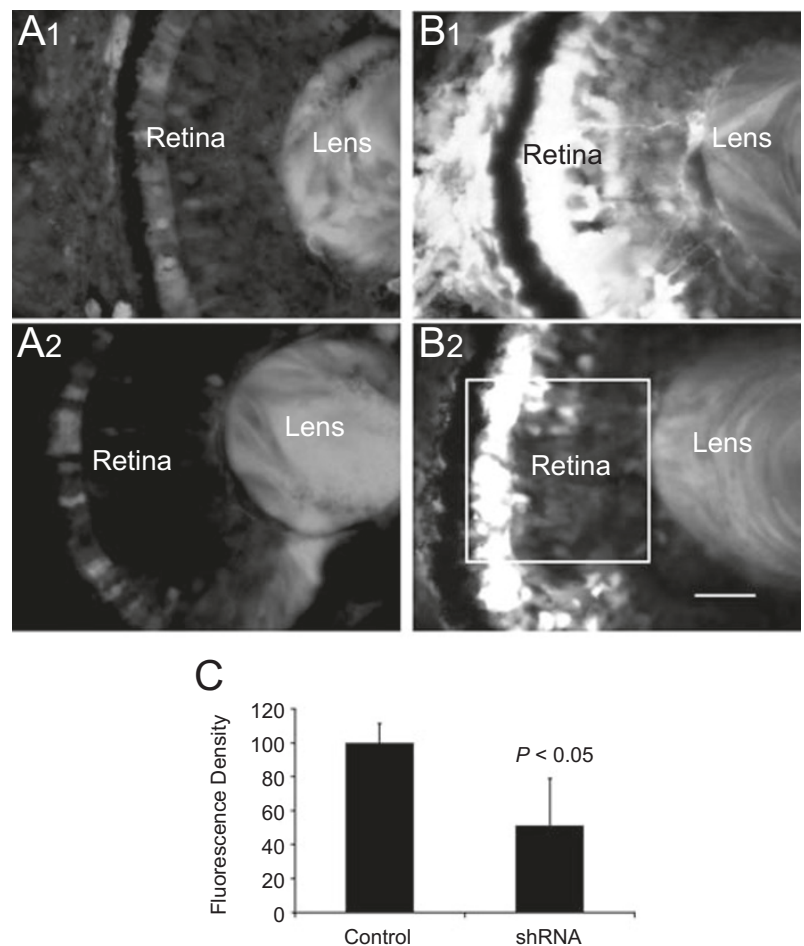

Figure 3 GFP expression in the retina was inhibited by shRNA transgenesis. Fluorescence photomicrographs of ocular cross sections revealed the decrease of GFP fluorescence from shRNA transgenic (A1, A2) and control embryos (B1, B2). (C) Fluorometric measurement of GFP intensity in retina sections was analyzed from St40/41 shRNA embryos $(n=7)$ and control embryos $(n=5)$. The area of measurement is shown by the $95 \times 95 \mu \mathrm{m}$ square in B2. Data were expressed in arbitrary fluorescence units. Each fluorescence value was normalized by first subtracting the autofluorescence present in non-GFP control animals and then comparing it to the level in the GFP-control animals, which was set at 100 . Scale bar for tissue sections in $\mathrm{B} 2=30 \mu \mathrm{m}$.

shRNA transgenic embryos was inhibited by $\sim 60 \%$ when compared with the control (Figure 4B, 4D). The reduction persisted in older tadpoles (St52 and St64) both at the GFP

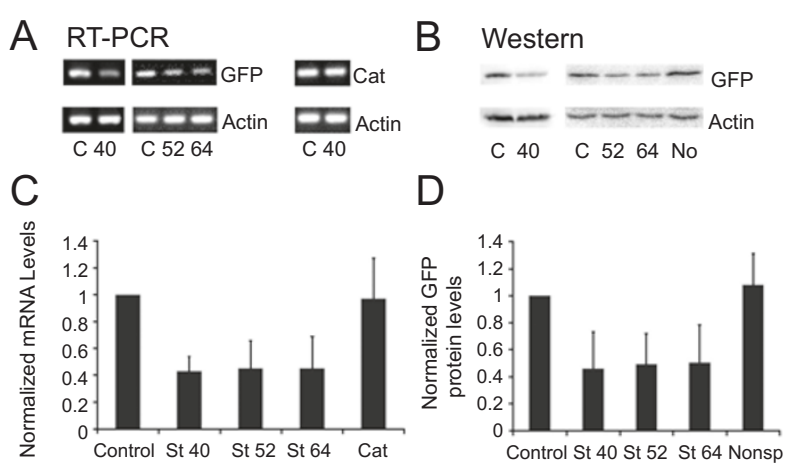

Figure 4 Transgene-driven RNAi knocks down GFP mRNA and protein expression in GFP transgenic embryos. mRNAs and proteins were extracted from individual animal. (A) mRNA levels in shRNA or control transgenic animals of stage (St) 40, 52 and 64 were analyzed using RT-PCR; (B) GFP protein expression was identified by Western blotting. (C) mRNA and (D) protein relative levels were measured for each sample and are shown as the average of animals $(n=7$ for St $40, n=5$ for St 52 and $n=3$ for St 64 ). $\beta$-actin was used as an internal control. The ratio of GFP and $\beta$-actin level was normalized to 1 in the control animals. The data are represented as mean \pm standard deviation. shRNA targeting GFP has no effect on $\beta$-catenin mRNA levels (A, C). Nonspecific shRNA (No in B, or Nonsp in D) has no effect on GFP protein expression. Abbreviations: C, control; 40, 52 and 64 are animal stages; Cat, $\beta$-catenin.

mRNA and protein levels (Figure 4). GFP shRNA did not inhibit mRNA levels of other genes (e.g., $\beta$-catenin, Figure 4A, 4C), and nonspecific GFP shRNA (targeting GFP in the same position, but with 3 mismatched nucleotides) had no effect on GFP mRNA levels in the GFP transgenic line (Figure 4B, 4D), demonstrating that the effects on GFP mRNA and protein were not caused by non-specific degradation of mRNA in the transgenic animals.

\section{Discussion}

One of the challenges of using $X$. laevis in developmental studies beyond gastrulation and early neurulation is the development of a reliable method to knock out or knock down specific genes. Gene expression can be inhibited at the mRNA levels in Xenopus via microinjection of antisense RNA, modified oligonucleotides or ribozyme, but that technique is restricted to the examination of early development events [22-24]. The rapidly developing RNAi technique has shown great promise in depressing gene expression in a variety of systems [8]. Of particular interest was the report that RNAi has been introduced successfully into mouse germline via transgenesis [25] and the study that H1-promoter-driven RNAi successfully recapitulated a genetic null phenotype in the mouse [26]. Compared with other popular species [9], there are limited reports 
on the application of RNAi in Xenopus. Microinjection of dsRNA into early Xenopus embryos has been shown to induce the inhibition of exogenous as well as endogenous gene expression $[13,14]$. Tissue-specific knock down of the expression of exogenous GFP has been shown via transgene-driven expression of long RNA duplexes (dsRNA) using the cardiac actin promoter [27].

Here we have demonstrated that transgene-driven RNAi could specifically and stably inhibit protein expression at later stages using a GFP transgenic line, suggesting that this approach may provide a useful tool to conduct genomics studies in Xenopus. A novel vector for gene silencing by vector-based RNAi in Xenopus was developed. This vector utilizes the $X$. tropicalis U6-promoter and the optimized short hairpin RNA structure followed by an RNA polymerase III termination signal. Xenopus U6, like mouse U6, consists of the distal and proximal sequence element (DSE and PSE) and a TATA box $[15,28]$. These elements appear to be species specific [29]. The effectiveness of the promoter was first validated in cultured Xenopus cells in vitro by inducing a knock down of GFP expression, which was followed by generating transgenic Xenopus embryos in vivo. $X$. tropicalis U6-promoter-driven shRNA against GFP was found to efficiently and specifically reduce the expression of GFP in a CMV-GFP transgenic Xenopus line, which contains one single integration site, at all the studied developmental stages (St 40-64). A reduction of $\sim 60 \%$ at both the mRNA and protein level were observed. However, no embryos were identified in which the RNAi completely inhibited GFP expression. In future studies we will examine whether the Xenopus U6 promoter together with the optimized short hairpin RNA structure may prove to be successful in suppressing endogenous target gene expression.

The level of GFP expression was reduced to $\sim 40 \%$ in the presence of U6-shRNA, but could not be completely eliminated. This may be due to a number of different reasons. First, the CMV promoter is a very efficient promoter in Xenopus, resulting in high levels of GFP expression [6]. Second, the Xenopus genome contains more than 500 copies of U6 genes, which may compete for the RNA polymerase III with U6-shRNA and cause low levels of shRNA expression. And finally, embryos will show differences in expression depending on the integration events. The integration events may vary from 1 to 5 , and each event may integrate 1 to 6 copies of shRNA per chromosomal location $[5,6]$. Thus, overall levels of shRNA may not be sufficient to knock out the targeted gene. On the other hand, as dsRNA or siRNAs result in non-specific degradation of RNA in mammalian cells [30,31], the low levels in shRNA expression are less likely to induce non-specific repression of gene expression in Xenopus.
Silencing endogenous genes in transgenics to produce obvious phenotypes is a challenge and one of the final goals of the studies. Dirks and coworkers (2003) reported that inhibition of endogenous target gene expression via transgene-driven RNAi has not yet been achieved; however, these experiments were performed using the human $\mathrm{H} 1$ promoter driving long inverted repeat transgenes, and tissue levels of mRNA and/or protein were not assessed. What is required to make RNAi technology successful in Xenopus? To effectively knock down genes and obtain phenotypes, we need to further examine the RNAi pathways in Xenopus. In particular, it needs to be determined whether all the known factors required for RNAi [32] are expressed in Xenopus and at what level, to identify which component(s) may be the limiting steps. In addition, it may be necessary to identify additional promoters whose activities are more tightly controlled in Xenopus, and/or to focus on genes that are not affected by the pseudo tetraploidy of $X$. laevis. Alternatively, it is likely that transgene-driven RNAi may make great progress in $X$. tropicalis or zebrafish due to their diploid genomes.

Based on the Xenopus studies with transgenesis and RNA silencing, a stable gene inhibition by transgene-driven RNAi in Xenopus may be practical and may provide a powerful alternative for 'loss of function' gene studies that have typically been performed in other vertebrate systems like the mouse. Although the transgenic mouse is an effective model for gene manipulation, it is expensive and time-consuming. On the other hand, hundreds of transgenic Xenopus embryos can be generated in one day. Furthermore, cell sizes of a number of different somatic cells are much larger in Xenopus than that in the mouse, and likewise, Xenopus cells are easier to maintain in culture. All these advantages make Xenopus more suitable for single cell analysis when compared to warm-blooded vertebrates. For example, the transgenic Xenopus model has been used successfully to investigate molecular mechanisms of photoreceptor degeneration or phototransduction at the single cell level $[33,34]$. If stable and heritable gene silencing can be achieved, transgene-driven RNAi could be a more favorable alternative to dominant negative, antisense and ribozyme technology to facilitate 'lose-of-function' studies in the Xenopus system. Combined with the traditional advantages of Xenopus as a developmental model, transgenesis and the ability to control gene expression spatially or temporally will revive Xenopus as one of the favorite models for functional genomics.

\section{Acknowledgements}

We would like to thank Dr Philippe Carbon (Institut de Biologie Moleculaire du CNRS, Strasbourg Cedex, France) 
for the generous gift of Xenopus tropicalis U6 gene, Drs Eddy De Robertis (University of California at Los Angeles) and Donald Brown (Carnegie Institution of Washington) for the gift of the male GFP transgenic frogs, and Dr Robert Grainger (University of Virginia), who introduced our laboratory to the transgenic technique. This work is supported by the National Institutes of Health / National Eye Institute EY 13728 (B.R.), EY 14793 (CORE); and an unrestricted grant from Research to Prevent Blindness (NY, NY) to MUSC. We would like to acknowledge the critical reading of the manuscript by Drs Kristen Kroll and Luanna Bartholomew.

\section{References}

1 Huang H, Marsh-Armstrong N, Brown DD. Metamorphosis is inhibited in transgenic Xenopus laevis tadpoles that overexpress type III deiodinase. Proc Natl Acad Sci U S A 1999; 96:962-7.

2 Hirsch N, Zimmerman LB, Gray J, et al. Xenopus tropicalis transgenic lines and their use in the study of embryonic induction. Dev Dyn 2002; 225:522-35.

3 Hartley KO, Nutt SL, Amaya E. Targeted gene expression in transgenic Xenopus using the binary Gal4-UAS system. Proc Natl Acad Sci U S A 2002; 99:1377-82.

4 Hawley SH, Wunnenberg-Stapleton K, Hashimoto C, et al. Disruption of BMP signals in embryonic Xenopus ectoderm leads to direct neural induction. Genes Dev 1995; 9:2923-35.

5 Kroll KL, Amaya E. Transgenic Xenopus embryos from sperm nuclear transplantations reveal FGF signaling requirements during gastrulation. Development 1996; 122:3173-83.

6 Marsh-Armstrong N, Huang H, Berry DL, Brown DD. Germ-line transmission of transgenes in Xenopus laevis. Proc Natl Acad Sci U S A 1999; 96:14389-93.

7 Hirsch N, Zimmerman LB, Grainger RM. Xenopus, the next generation: $X$. tropicalis genetics and genomics. Dev Dyn 2002; 225:422-33.

8 Hannon GJ. RNA interference. Nature 2002; 418:244-51.

9 Thakur A. RNA interference revolution. Electronic J Biotechnol 2003; 6:39-49.

10 Gou D, Jin N, Liu L. Gene silencing in mammalian cells by PCR-based short hairpin RNA. FEBS Lett 2003; 548:113-8.

11 Abbas-Terki T, Blanco-Bose W, Deglon N, Pralong W, Aebischer P. Lentiviral-mediated RNA interference. Hum Gene Ther 2002; 13:2197-201.

12 Shen C, Buck AK, Liu X, Winkler M, Reske SN. Gene silencing by adenovirus-delivered siRNA. FEBS Lett 2003; 539:111-4.

13 Zhou Y, Ching YP, Kok KH, Kung HF, Jin DY. Post-transcriptional suppression of gene expression in Xenopus embryos by small interfering RNA. Nucleic Acids Res 2002; 30:1664-9.

14 Nakano H, Amemiya S, Shiokawa K, Taira M. RNA interference for the organizer-specific gene Xlim-1 in Xenopus embryos. Biochem Biophys Res Commun 2002; 274:434-9.

15 Krol A, Carbon P, Ebel JP, Appel B. Xenopus tropicalis U6 snRNA genes transcribed by Pol III contain the upstream promoter elements used by Pol II dependent U snRNA genes. Nucleic Acids Res 1987; 15:2463-78.

16 Amsterdam A, Lin S, Moss LG, Hopkins N. Requirements for green fluorescent protein detection in transgenic zebrafish embryos. Gene 1996; 173:99-103.

17 Sakaguchi DS, Moeller JF, Coffman CR, Gallenson N, Harris WA. Growth cone interactions with a glial cell line from embryonic Xenopus retina. Dev Biol 1989; 134:158-74.

18 Mitas M, Mikhitarian K, Walters C, et al. Quantitative real-time RT-PCR detection of breast cancer micrometastasis using a multigene marker panel. Int J Cancer 2001; 93:162-71.

19 Sui G, Soohoo C, Affar el B, Gay F, et al. A DNA vector-based RNAi technology to suppress gene expression in mammalian cells. Proc Natl Acad Sci U S A 2002; 99:5515-20.

20 Brummelkamp TR, Bernards R, Agami R. A system for stable expression of short interfering RNAs in mammalian cells. Science 2002; 296:550-3.

21 Amaya E, Kroll KL. A method for generating transgenic frog embryos. Methods Mol Biol 1999; 97:393-414.

22 Lim W, Furlow JD. Ribozyme suppression of endogenous thyroid hormone receptor activity in Xenopus laevis cells. Nucleic Acids Res 2002; 30:3490-6.

23 Heasman J, Kofron M, Wylie C. Beta-catenin signaling activity dissected in the early Xenopus embryo: a novel antisense approach. Dev Biol 2000; 222:124-34.

24 Nutt SL, Bronchain OJ, Hartley KO, Amaya E. Comparison of morpholino based translational inhibition during the development of Xenopus laevis and Xenopus tropicalis. Genesis 2001; 30:110-3.

25 Hasuwa H, Kaseda K, Einarsdottir T, Okabe M. Small interfering RNA and gene silencing in transgenic mice and rats. FEBS Lett 2002; 532:227-30.

26 Kunath T, Gish G, Lickert H, et al. Transgenic RNA interference in ES cell-derived embryos recapitulates a genetic null phenotype. Nat Biotechnol 2003; 21:559-61.

27 Dirks RP, Bouw GB, Huizen RR, Jansen EJ, Martens JM. Functional genomics in Xenopus laevis: Towards transgene-driven RNA interference and cell-specific transgene expression. Current Genomics 2003; 4:699-711.

28 Carbon P, Murgo S, Ebel JP, et al. A common octamer motif binding protein is involved in the transcription of U6 snRNA by RNA polymerase III and U2 snRNA by RNA polymerase II. Cell 1987; 51:71-9.

29 Das G, Henning D, Reddy R. Structure, organization, and transcription of Drosophila U6 small nuclear RNA genes. J Biol Chem 1987; 262:1187-93.

30 Leaman DW, Salvekar A, Patel R, Sen GC, Stark GR. A mutant cell line defective in response to double-stranded RNA and in regulating basal expression of interferon-stimulated genes. Proc Natl Acad Sci U S A 1998; 95:9442-7.

31 Persengiev SP, Zhu X, Green MR. Nonspecific, concentration-dependent stimulation and repression of mammalian gene expression by small interfering RNAs (siRNAs). RNA 2004; 10:12-8.

32 Denli AM, Hannon GJ. RNAi: an ever-growing puzzle. Trends Biochem Sci 2003; 28:196-201.

33 Kefalov V, Fu Y, Marsh-Armstrong N, Yau KW. Role of visual pigment properties in rod and cone phototransduction. Nature 2003; 425:526-531.

34 Jin S, Cornwall MC, Oprian DD. Opsin activation as a cause of congenital night blindness. Nat Neurosci 2003; 6:731-5.

Edited by Yun-Bo Shi 